\title{
STATE-TRAJECTORY BEHAVIOR IN HIGH-ORDER, LOWPASS SIGMA-DELTA MODULATORS WITH DISTINCT NTF ZEROS
}

\author{
Ngai Wong and Tung-Sang Ng \\ Department of Electrical and Electronic Engineering, \\ The University of Hong Kong, Pokfulam Road, Hong Kong. \\ nwong@eee.hku.hk, tsng@eee.hku.hk \\ Tel.: ++ $852+28578406$, Fax: ++ $852+25598738$
}

\begin{abstract}
This paper presents a generic, scalable approach to obtain closed-form state-trajectory expressions for highorder (order $>2$ ) lowpass sigma-delta $(\Sigma \Delta)$ modulators with distinct noise transfer function (NTF) zeros. Constant modulator input is assumed. The techniques of state-space diagonalization, continuous-time embedding, and Poincaré map analysis are combined and extended. It is shown that an even-order modulator can be decomposed into individual second-order subsystems with circular trajectories about two half-plane centers, while an odd-order modulator will result in an additional first-order subsystem represented by an oscillating quantity. The trajectory and half-plane transition expressions thus obtained provide effective tools for stability analysis of $\Sigma \Delta$ modulators.
\end{abstract}

\section{INTRODUCTION}

Sigma-delta $(\Sigma \Delta)$ modulation $[1]-[3]$ is a popular and increasingly important technique that seamlessly bridges continuous and digital domains by the use of oversampling and noise shaping. Single-bit modulators are particularly appealing due to their inherent linearity and high tolerance of analog component imperfections. Theoretical development and understanding of $\Sigma \Delta$ modulators, however, have been far lagging behind. Stability remains one long-standing major issue that thrcatens the use of high-order $\Sigma \Delta$ modulators capable of providing high signal-to-noise ratio (SNR). Linearized analyses (e.g., [4], [5]) of stability problem suffer from their approximation nature and inadequacy to explain intricate phenomena such as chaos and limit cycles. While nonlinear dynamical approaches (e.g., [6][9]), though being exact, are usually complicated and restricted to second-order modulators.

Section 2 of this paper extends the state-space diagonalization method of Stciner and Yang [10] to cover the case of transition matrices having complex conjugate eigenvalues, which is commonly found in $\Sigma \Delta$ modulators with distinct (e.g., optimized) NTF zeros [3]. Section 3 introduces a generic continuous-time cmbedding [7] procedure and shows that this class of high-order $\Sigma \Delta$ modulators can be decomposed into second-order and first-order subsystems. Assuming constant modulator input, closed-form and scalable state-trajectory expressions are derived for them. Section 4 studies the transition across the hyperplane that divides the modulator dynamics into two linear halfplanes. Formulae for the generalized Poincaré sections are also given. Altogether these expressions constitute effective tools for DC stability analysis of $\Sigma \Delta$ modulators. Finally, Section 5 draws the conclusion.

\section{SIMILARITY TRANSFORMS}

This section describes the diagonalization of an arbitrary-order $\Sigma \Delta$ modulator with distinct NTF zeros. The cascade-of-resonators architecture [5] (Fig. 1a) is chosen for illustration due it its popularity. For brevity, 4 th and 5 th-order modulators are considered but how the formulation can be adapted to higher order systems should be obvious from the context. Using a similar approach as in [11], by defining the state-vector $\mathbf{x}^{(0)}=\left[x_{1}^{(0)} \cdots x_{N}^{(0)}\right]$ and $\rho=z-1$, the state-space format of a parameterized $N$ th-order modulator is

$$
\left\{\begin{array}{l}
\rho \mathbf{x}^{(0)}=\mathbf{\Lambda}_{\mathbf{z}}^{(0)} \mathbf{x}^{(0)}+\mathbf{b}_{\mathbf{z}}^{(0)} u+\mathbf{a}_{\mathbf{z}}^{(0)} v \\
y=b_{0} u+\mathbf{d}_{\mathbf{z}}^{(0)} \mathbf{x}^{(0)} \\
v=\operatorname{sgn}(y)
\end{array},\right.
$$

specifically for our example in Fig. 1a ( $N=4$ and 5),

$\mathbf{x}^{(0)}=\left[\begin{array}{lllll}x_{1}^{(0)} & x_{2}^{(0)} & x_{3}^{(0)} & x_{4}^{(0)} & \mid x_{3}^{(0)}\end{array}\right]^{T}$.

$\mathbf{b}_{2}^{(n)}=\left[\begin{array}{llllll}b_{1} k_{1} & b_{2} k_{1} k_{2} & b_{3} k_{1} k_{2} k_{3} & b_{4} k_{1} \cdots k_{4} & \mid b_{5} k_{1} \cdots k_{5}\end{array}\right]^{T}$.

$\mathbf{a}_{x}^{(0)}=-\left[\begin{array}{llllll}a_{1} k_{1} & a_{2} k_{1} k_{2} & a_{3} k_{1} k_{2} k_{3} & a_{4} k_{1} \cdots k_{4} & \mid a_{5} k_{1} \cdots k_{3}\end{array}\right]^{\prime}$.

$\mathbf{d}_{z}^{(0)}=\left[\begin{array}{lllll}k_{1}^{-1} & 0 & 0 & 0 & 0\end{array}\right]^{\top}$.

$\mathbf{A}_{x}^{(0)}=\mathbf{A}_{1}+z \mathbf{A}_{2}$

$=\left[\begin{array}{ccccc}0 & 0 & 0 & 0 & 0 \\ -r_{1} k_{2} & 0 & k_{3}^{-1} & 0 & 0 \\ 0 & 0 & 0 & 0 & 0 \\ 0 & 0 & -r_{2} k_{4} & 0 & k_{s}^{-1} \\ \hline 0 & 0 & 0 & 0 & 0\end{array}\right]+z\left[\begin{array}{cccc|c}0 & k_{2}^{-1} & 0 & 0 & 0 \\ 0 & 0 & 0 & 0 & 0 \\ 0 & 0 & 0 & k_{4}^{-1} & 0 \\ 0 & 0 & 0 & 0 & 0 \\ \hline 0 & 0 & 0 & 0 & 0\end{array}\right]$.

Here $\operatorname{sgn}(y)$ is the sign function whose output is +1 when $y \geq 0$ and -1 when $y<0$. The bracketed superscripts denote the number of similarity transforms the statevectors or matrices have undergone. Note that (2) depicts the case of an odd-order system $(N=5)$. For an even-order modulator $(N=4)$, the elements outside the delimiting lines are simply dropped, e.g., the rightmost column and bottommost row in $\mathbf{A}_{x}^{(i)}$, the rightmost element in $\mathbf{x}^{(0)}$ etc. This convention of combined representation of even and odd-order systems will be followed throughout this paper. Next, by substiluting $\rho=z-1$ and considering the special structure of $\mathbf{A}_{2}$, the first equation of $(1)$ is rewritten as $z \mathbf{x}^{(i)}=\left(\mathbf{I}+\mathbf{A}_{2}\right)\left(\mathbf{I}+\mathbf{A}_{1}\right) \mathbf{x}^{(\mathbf{i})}+\left(\mathbf{I}+\mathbf{A}_{2}\right) \mathbf{b}_{2}^{(1) \prime} u+\left(\mathbf{I}+\mathbf{A}_{2}\right) \mathbf{a}_{2}^{(0)} v$.

Examining Fig. 1a, the NTF zeros are just the poles of the transfer function from $v$ to $y$ with zero input $(u=0)$. Thus $\left(\mathbf{I}+\mathbf{A}_{2}\right)\left(\mathbf{I}+\mathbf{A}_{1}\right)$ must have eigenvalues corresponding to the distinct NTF zeros and is diagonalizable by a similarity transform through a nonsingular matrix $\mathbf{T}_{\mathbf{d}}$, resulting in 


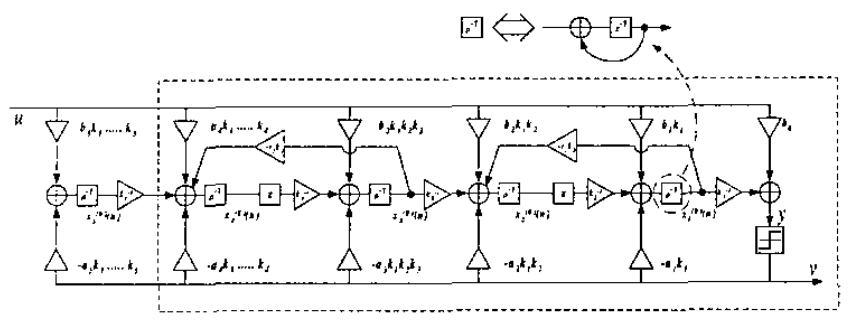

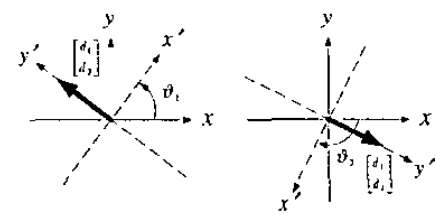

Fig. I. (a) Example $\Sigma \Delta$ modulators (5th-order as a whole and 4th-order within the dotted line) in a parameterized cascade-of-resonator structure; $(b)$ the normalization process by rotating to the new coordinate system $x^{\prime}, y^{\prime}$.

$$
\begin{aligned}
& \left\{\begin{array}{l}
z \mathbf{x}^{(1)}=\mathbf{A}_{z}^{(1)} \mathbf{x}^{(1)}+\mathbf{b}_{z}^{(1)} u+\mathbf{a}_{2}^{(1)} v \\
y=b_{0} u+\mathbf{d}_{z}^{(1)} \mathbf{x}^{(1)}
\end{array}\right. \\
& \text { - where } \\
& x^{(1)}=T_{d}^{-1} x^{(0)}, b_{2}^{(1)}=T_{d}^{-1}\left(I+A_{2}\right) b_{d}^{(a)}, a_{x}^{(1)}=T_{d}^{-1}\left(I+A_{2}\right) a_{x}^{(0)}, \\
& d_{2}^{(1)}=d_{2}^{(1)} T_{d}, A_{2}^{(1)}=T_{d}^{-1}\left(I+A_{2}\right)\left(I+A_{1}\right) T_{d} \\
& =\left[\begin{array}{ccc|c}
\mathbf{e}_{1} & & 0 & 0 \\
& \ddots & & \vdots \\
0 & & \mathbf{e}_{S_{2}} & 0 \\
\hline 0 & \cdots & 0 & 1
\end{array}\right], \quad \mathbf{e}_{i}=\left[\begin{array}{cc}
e^{\mu \omega_{i}} & 0 \\
0 & e^{-j e_{i}}
\end{array}\right] .
\end{aligned}
$$

Here $N_{2}=\lfloor N / 2\rfloor$ is the number of second-order subsystems. The bolded $\mathbf{0} \mathrm{s}$ stand for zeros apart from the diagonal. The $\omega_{i} \mathrm{~s}$ (by convention $\omega_{i}>0$ ) correspond to the distinct NTF zero frequencies. It can be verified that the elements in other state-matrices are also complex conjugate pairs. To visualize the dynamics it is desirable to work with real state-variables. This is achieved by a similarity transform that separates the real and imaginary parts of the conjugate elements, namely,

$$
\boldsymbol{T}_{\mathbf{R}}=\left[\begin{array}{ccc|c}
\mathbf{\Omega} & & \mathbf{0} & 0 \\
& \ddots & & \vdots \\
\mathbf{0} & & \mathbf{\Omega} & 0 \\
\hline 0 & \cdots & 0 & 1
\end{array}\right] \quad \mathbf{\Omega}=\left[\begin{array}{cc}
1 & j \\
1 & -j
\end{array}\right]
$$

Such transform produces

$$
\left\{\begin{array}{l}
z^{(2)}=\Lambda_{z}^{(2)} \mathbf{x}^{(2)}+\mathbf{b}_{x}^{(2)} u+\mathbf{a}_{x}^{(2)} v \\
y=b_{0} u+\mathbf{d}_{2}^{(2)} \mathbf{x}^{(2)}
\end{array}\right.
$$

with

$$
\mathbf{x}^{(2)}=\mathbf{T}_{\pi}^{-1} \mathbf{x}^{(1)}, \mathbf{b}_{a}^{(2)}=\mathbf{T}_{\pi}^{-1} \mathbf{b}_{a}^{(1)}, \mathbf{a}_{2}^{(2)}=\mathbf{T}_{-\pi}^{-1} \mathbf{a}_{2}^{(1)}, \mathbf{d}_{\alpha}^{(2)}=\mathbf{d}_{a}^{(1)} \mathbf{T}_{\pi}
$$$$
A_{2}^{(2)}=T_{*}^{-i} A_{z}^{(1)} T_{* 3}
$$$$
=\left[\begin{array}{ccc|c}
\Theta_{1} & & 0 & 0 \\
& \ddots & & \vdots \\
0 & & \boldsymbol{\Theta}_{N_{2}} & 0 \\
\hline 0 & \cdots & 0 & 1
\end{array}\right], \quad \Theta_{i}=\left[\begin{array}{cc}
\cos \omega_{i} & -\sin \omega_{i} \\
\sin \omega_{i} & \cos \omega_{i}
\end{array}\right]
$$

The elements in all state-matrices are made real by this transformation. Now the state-space representation is transformed into parallel second-order subsystems (plus an additional first-order subsystem for odd-order modulators). In this block-diagonal form, the statevariables of every subsystem become almost decoupled, interacting only through the quantizer, function $v=\operatorname{sgn}(y)$. By detailing the second equation in (7),

$$
\begin{aligned}
y=b_{0} u & +\left[\begin{array}{ll}
d_{1} & d_{2}
\end{array}\right]\left[\begin{array}{ll}
x_{1}^{(2)} & x_{2}^{(2)}
\end{array}\right]^{T}+\cdots \\
& +\left.\left[\begin{array}{ll}
d_{2 N_{2}-1} & d_{2 N_{1}}
\end{array}\right]\left[\begin{array}{ll}
x_{2 N_{2}}^{(2)} & x_{2 N_{2}}^{(2)}
\end{array}\right]^{7}\right|^{+}+d_{2 w_{2}+1} x_{2 N_{1}+1}^{(2)},
\end{aligned}
$$

these summation terms are in fact dot products which represent the projections of the state-variables of cvery second-order subsystem onto the vectors formed by

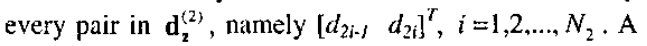
further transformation and simplification is to rotate and scalc the coordinate system of every second-order subsystem such that these vectors become the unit vectors in the conventional upward sense (see Fig. 1b), and to scale $d_{2 N_{2}+1}$ to unity for odd-order modulators. This process, denoted as the normalization process, is done by the normalization matrix $T_{n}$ defined as

$$
\mathrm{r}_{\mathrm{a}}=\left[\begin{array}{ccc|c}
\mathrm{n}_{1} & & 0 & 0 \\
& \ddots & & \vdots \\
0 & & \mathrm{n}_{\mathrm{s}_{1}} & 0 \\
\hline 0 & \cdots & 0 & d_{2 N_{2}+1}^{-1}
\end{array}\right], \quad \mathbf{n}_{i}=\left(d_{2 i-1}^{2}+d_{21}^{2}\right)^{-1 / 2}\left[\begin{array}{cc}
\cos \vartheta_{1} & -\sin \vartheta_{i} \\
\sin \theta_{1} & \cos \theta_{i}
\end{array}\right]
$$

where $\vartheta_{i} \mathrm{~s}$ are as determined in Fig. $1 \mathrm{~b}$. Notice that the transformation by $\boldsymbol{T}_{\mathbf{n}}$ has no effect on $\mathbf{A}_{\mathbf{z}}^{(2)}$ because only the coordinate system of every second-order subsystem is changed but not its nature. Summarizing,

$$
\left\{\begin{array}{l}
z \mathbf{x}^{(3)}=\mathbf{A}_{z}^{(3)} \mathbf{x}^{(3)}+\mathbf{b}_{z}^{(3)} u+\mathbf{a}_{z}^{(3)} v \\
y=b_{v} u+\mathbf{d}_{z}^{(3)} \mathbf{x}^{(3)}
\end{array}\right.
$$

where

$$
\begin{aligned}
& \mathbf{x}^{(3)}=\mathbf{T}_{n}^{-1} \mathbf{x}^{(2)}, \quad \mathbf{b}_{2}^{(3)}=\mathbf{T}_{n}^{-1} \mathbf{b}_{z}^{(2)}, \\
& \mathbf{a}_{2}^{(3)}=\mathbf{T}_{1}^{-1} \mathbf{a}_{x}^{(2)}, \quad \mathbf{A}_{2}^{(3)}=\mathbf{T}_{n}^{-1} \mathbf{A}_{2}^{(2)} \mathbf{T}_{n}=\mathbf{A}_{2}^{(2)} \\
& \mathbf{d}_{2}^{(3)}=\mathbf{d}_{x}^{(2)} \mathbf{T}_{n}=\left[\begin{array}{lllllll}
0 & 1 & \cdots & 0 & 1 & 1
\end{array}\right] .
\end{aligned}
$$

It should be stressed that (1), (4), (7) and (11) all describe the same $\Sigma \Delta$ modulator because $y$ (thus the modulator output $v$ ) is invariant. The only distinction lies in the different choices of state-vectors.

\section{CONTINUOUS-TIME EMBEDDING}

Embedding [7] refers to the construction of a set of continuous differential equations whose solution, in the form of a time-evolving trajectory, contains every point along the discrete trajectory solved from the discrete system of equations. Such process results in continuoustime functions easier to handle analytically. To embed an arbitrary-order discrete-time system like (11), we consider a continuous-time counterpart

$$
\left\{\begin{array}{l}
\dot{\mathbf{x}}_{\mathbf{c}}=\mathbf{A}_{\mathbf{c}} \mathbf{x}_{\mathbf{c}}+\mathbf{b}_{\mathbf{c}} u+\mathbf{a}_{\mathbf{c}} v \\
y=b_{0} u+\mathbf{d}_{\mathbf{c}} \mathbf{x}_{\mathbf{c}}
\end{array}\right.
$$

where the subscript $c$ stands for continuous-time. Assuming $t \geq k$ and $\mathbf{x}_{\mathbf{c}}(k)$ is known, the exact solution of the first equation in (13) is $\mathbf{x}_{\mathrm{c}}(t)=\exp \left(\mathrm{A}_{\mathrm{c}}(t-k)\right) \mathbf{x}_{\mathrm{c}}(k)+\int_{k}^{t} \exp \left(\mathbf{A}_{\mathrm{c}}(t-\tau)\right)\left[\mathbf{b}_{\mathrm{c}} u(\tau)+\mathbf{a}_{\mathrm{c}} v(\tau)\right] d \tau$ 
where $\exp (0)$ stands for matrix exponential. Now assume $u$ and $v$ remain constant during the time interval $k \leq t \leq k+1$, then at the next time instance

$$
\begin{aligned}
\mathbf{x}_{\mathrm{c}}(k+1) & =\operatorname{cxp}\left(\mathbf{A}_{\mathrm{c}}\right) \mathbf{x}_{\mathrm{c}}(k)+\int_{k}^{k+1} \exp \left(\mathbf{A}_{\mathbf{c}}(k+1-\tau)\right)\left[\mathbf{b}_{\mathrm{c}} u(\tau)+\mathbf{a}_{\mathrm{c}} v(\tau)\right] d \tau \\
& =\exp \left(\boldsymbol{A}_{\mathrm{c}}\right) \mathbf{x}_{\mathrm{c}}(k)+\left[\int_{0}^{1} \operatorname{cxp}\left(\boldsymbol{A}_{\mathrm{c}} \tau\right) d \tau\right]\left[\mathbf{b}_{\mathrm{c}} u(k)+\mathbf{a}_{\mathrm{c}} v(k)\right]
\end{aligned}
$$

Mapping (15) to (11), it follows that

$$
A_{\mathrm{c}}=\ln \left(\mathrm{A}_{2}^{(3)}\right)=\left[\begin{array}{ccc|c}
\mathbf{w}_{1} & & 0 & 0 \\
& \ddots & & \vdots \\
0 & & \mathbf{w}_{\alpha_{2}} & 0 \\
\hline 0 & \cdots & 0 & 0
\end{array}\right] . \quad \mathbf{w}_{t}=\left[\begin{array}{cc}
0 & -\omega_{i} \\
\omega_{i} & 0
\end{array}\right] .
$$

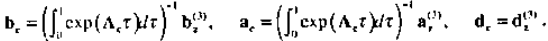

Now we have established a relationship between the discrete-time and continuous-time models. According to (15), a unit time-step advancement (i.e., increasing $t$ by 1) in the continuous-time trajectory corresponds to a hop to the next state in the discrete-time domain. For case of exposition we define $\mathbf{a}_{\mathrm{c}}=\left|\alpha_{1} \cdots \alpha_{2 N_{2}}\right| \alpha_{2 N_{2}+1} \mid$, $\mathbf{b}_{\mathbf{c}}=\left[\beta_{1} \cdots \beta_{2 N_{2}} \mid \beta_{2 N_{2}+1}\right]$. Also, we denote a matrix or vector after dropping the terms outside the delimiters by a tilde sign, e.g., in (16), $\overline{\mathbf{A}}_{\mathrm{c}}=\operatorname{diag}\left[\mathbf{w}_{1} \cdots \mathbf{w}_{N_{2}}\right]$ and $\overline{\mathbf{b}}_{\mathrm{c}}$ is $\mathbf{b}_{\mathbf{c}}$ without the last element etc. It should stressed that this tilde sign is immaterial for even-order modulators wherein $\mathbf{A}_{\mathbf{c}}=\overline{\mathbf{A}}_{\mathbf{c}}, \quad \mathbf{b}_{\mathbf{c}}=\tilde{\mathbf{b}}_{\mathrm{c}}$ etc. For a constant $u$, a further simplification is possible by defining another continuous-time state-vector $\mathbf{x}(t)$ which is a translation of $x_{c}(t)$. given by

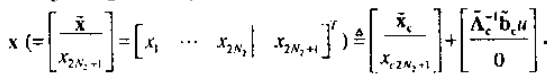

Ultimately (13) becomes

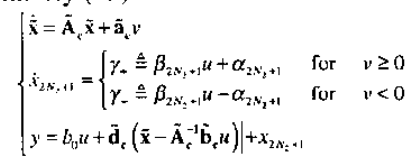

where the middle line in (18) is only for odd-order modulators. Equation (18) can be easily solved to give

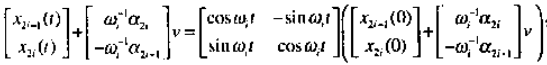

$i=1,2, \ldots, N_{2}$. And for the possible first-order subsystem

$$
x_{2 N_{*}, 1}(t)=\left\{\begin{array}{lll}
x_{2 N_{2+1}}(0)+\gamma_{+} t & \text { for } & \nu \geq 0 \\
x_{2 N_{t}+1}(0)+\gamma_{-} t & \text { for } & \nu<0
\end{array} .\right.
$$

These two equations reveal that second-order subsystems represents circular trajectories orbiting about two centers symmetric about the origin; while the first-order subsystem is an oscillating quantity. Fig. 2 shows the typical dynamics of these subsystems. Recalling that every unit time-step advancement in (19) \& (20) corresponds to a hop to the nexi state for a discrete trajectory, continuous-lime embedding actualiy "fills in" the states between consecutive discrete states by assuming continuous (infinitesimal) cvolution of trajectory. Consequently, by restricting $t$ to be nonnegative integers, (18)-(20) can also be used to describe the discrete trajectory provided the starting condition $\mathbf{x}(0)$ in a half-plane is known.

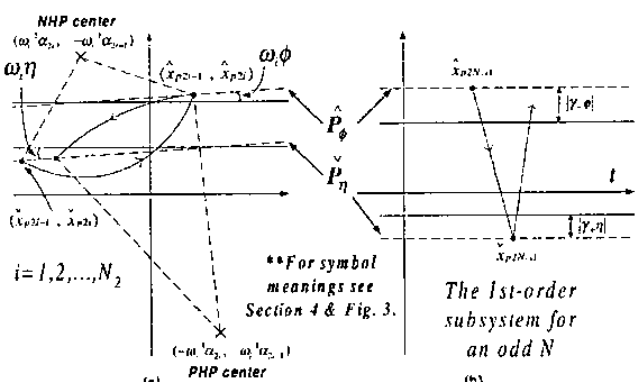

Fig. 2. Typical decomposed subsystems: (a) secondorder (positions of half-plane centers can vary as long as they are symmetric about the origin); (b) first-order.
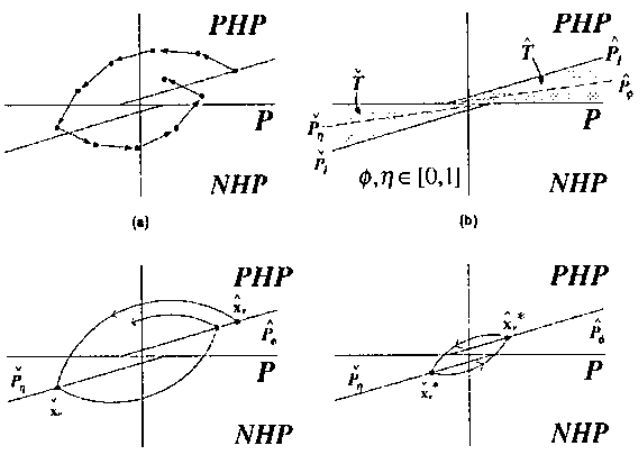

Fig. 3. Conceptual drawings of nonlinear dynamics (a) discrete trajectory; (b) transition wedges and Poincaré sections; (c) continuous trajectory; (d) fixed-points and the corresponding linit cycle.

\section{POINCARÉ SECTIONS}

The third line in (18) can be verified to be equal to

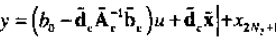

$$
\begin{aligned}
& =\left(b_{\mathrm{q}}+\tilde{\mathbf{d}}_{\mathbf{z}}\left(\tilde{\mathbf{I}}-\tilde{\mathbf{A}}_{z}\right)^{-1} \tilde{\mathbf{b}}_{\mathbf{x}}\right) u+\tilde{\mathbf{d}}_{\mathrm{c}} \overline{\mathbf{x}} \mid+x_{2 x_{z}+1} \\
& =K u+\sum_{i=1}^{N_{i}} x_{21} \mid+x_{2 w_{j}+1}
\end{aligned}
$$

where $\tilde{\mathbf{I}}$ is an identity matrix with appropriate dimension. In the second line of (21) the superscripts for the discrete system matrices are omilted because the cxpression inside the bracket is invariant under similarity transforms. This bracketed value, denoted by $K$, is in fact the forward-path resonator DC gain (ignoring the last branch in odd-order modulators which is not part of a resonator, i.c., by putting $b_{5} k_{1} \cdots k_{5}=0$ in Fig. la) from $u$ to $y$ with $v=0$. Therefore, we have the hyperplane $\boldsymbol{P}\left(K u+\sum_{i=1}^{N_{2}} x_{2 i} \mid+x_{2 N_{2}+1}=0\right)$ that divides the state-space into two regions, called the positive halfplanc $\boldsymbol{P H P}\left(\Sigma_{i=1}^{N_{2}} x_{2 i} I+x_{2 N_{2}+1} \geq-K u\right)$ and the negative half-plane NHP $\left(\sum_{i=1}^{N_{2}} x_{2 i} \mid+x_{2 N_{2}+1}<-K u\right)$ wherein the quantizer output $v$ is +1 and -1 respectively. This demonstrates that the transform in (10) is essential for visualization because now the half-plane conditions can readily be told by summing the "y-coordinates" of all 


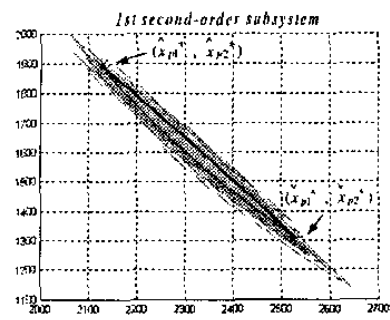

(a)

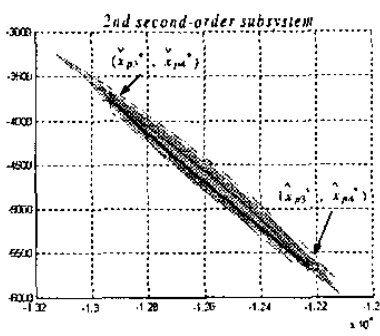

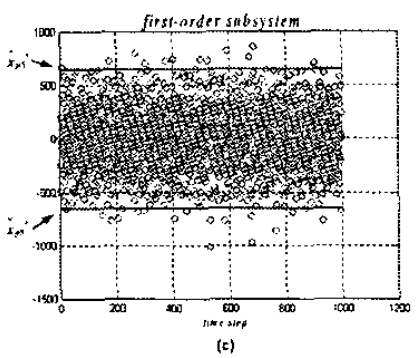

(c)

Fig. 4(a)-(c) Simulation of a 5th-order $\Sigma \Delta$ modulator with optimized NTF zeros under stable operation $(u=0.48$, $O S R=64,1000$ runs) together with the boundary transition flow limit cycle denoted by bolded lines.

subsystems. Employing the convention of Wang [7], mode- $\wedge$ and mode- $\vee$ are used to distinguish the positive and negative states of the quantizer output. A practical $\Sigma \Delta$ modulator is a sampled-data system and its state trajectory undergoes discrete mapping as in Fig. 3a. The discrete constraint will carry the trajectory, after hitting $\boldsymbol{P}$, somewhere beyond (and including) $\boldsymbol{P}$ where it stays for one time instance (known as the transition point [7]) before changing into dynamics of the opposite halfplane. The two wedges shown in Fig. 3b, bounded by $\boldsymbol{P}$ and the far end boundaries $\hat{P}_{1}$ and $\dot{P}_{1}$, denote the possible regions where the transition points occur. These wedges are called the positive and negative transition wedges $\hat{T}$ and $\stackrel{\leftarrow}{T}$. In reality the transition may take place anywhere on the chosen Poincaré sections [7] $\hat{P}_{*}$ and $\check{P}_{\eta}$, where $\phi, \eta \in[0,1]$ are called the $\hat{T}$ and $\stackrel{\sim}{T}$ tilt factors for obvious reasons. Their equations are obtained by realizing the fact that when states residing on $\hat{P}_{p}$ and $\check{P}_{\eta}$ (i.c., $\hat{\mathbf{x}}_{\mathrm{p}}$ and $\grave{\mathrm{x}}_{\mathrm{p}}$ in Fig. $3 \mathrm{c}$ ) are mapped by $\phi$ and $\eta$ time-step backward, they will land on $P$, so

$$
\begin{aligned}
& \hat{P}_{\phi}:\left(\sum_{i=1}^{N_{i}}-\left(\hat{x}_{\rho 2 i-1}-\omega_{i}^{-1} \alpha_{2 i}\right) \sin \omega_{\phi} \phi\right. \\
& \left.+\left(\hat{x}_{p i i}+\omega_{i}^{-1} \alpha_{2 i-1}\right) \cos \omega_{i \phi}-\omega_{i}^{-1} \alpha_{2 i-1}\right) \mid+\hat{x}_{m V}-\gamma_{-\phi}=-K_{i}
\end{aligned}
$$

and

$$
\begin{aligned}
& \check{P}_{\eta}:\left(\sum_{i=1}^{N_{3}}-\left(\check{x}_{p 2 i-1}+\omega_{i}^{-1} \alpha_{2 i}\right) \sin \omega_{i} \eta\right. \\
& \left.\left.+\left(\tilde{x}_{p 2 i}-\omega_{i}^{-1} \alpha_{2 i-1}\right) \cos \omega_{i} \eta+\omega_{i}^{-1} \alpha_{2 i-1}\right)\right)+\dot{x}_{p i v}-\gamma . \eta=-K u .
\end{aligned}
$$

The boundedness and positioning (and thus the modulator stability and cxact values of $\phi$ and $\eta$ ) of the transition points into these wedges are, however, analytically intractable. Fig. 3d shows the possible continuous-time first-retum limit cycle and fixed-points, denoted by $\hat{\mathbf{x}}_{\mathbf{p}}{ }^{*}$ and $\mathrm{x}_{\mathbf{p}}{ }^{*}$, arising from a particular transition flow. Wang has investigated the case of $\phi=\eta=$ 1 , called the boundary transition flow, which has intuitively further stretched limit cycles (but whose stability do not necessarily guarantee stability of a $\Sigma \Delta$ modulator). Fig. 4 shows the actual discrete trajectories transformed into the subsystem framework, together with the limit cycle of the boundary transition flow. In a recent work by the authors [12], it is shown that stability analysis must involve all possible transition flows. By virtue of the trajectory and transition expressions presented in this paper, cfficient numerical methods can be used to locate all possible fixed-points and evaluate the stability of their accompanying limit cycles [12]

\section{CONCLUSION}

This paper has presented a nonlinear dynamical approach to the investigation of the state-trajectory behavior of high-order (order $>2$ ), lowpass $\Sigma \Delta$ modulators with distinct NTF zeros under constant input. Algebraic difficulties arising from the nonlinear quantizer function have been tackled by combining and extending the techniques of state-space diagonalization, continuous-time embedding and Poincaré map analysis. It has been shown that this class of $\Sigma \Delta$ modulators can be decomposed into individual second-order subsystems with circular trajectories orbiting about two half-plane centers, whereas for odd-order modulators there is an additional first-order subsystem exhibiting an oscillating quantity. Trajectory and transition expressions thus derived constitute useful tools for stability analysis.

\section{REFERENCES}

[1] J. C. Candy and G. C. Temes, Lds., Oversampling Delta-Sigma Data Converters. New York: IEEE Press, 1992.

[2] S. R. Norsworhy, R. Schreier, and G. C. Temes, Delia-Sigma Data Converters Ncw York: ICEE Press, 1997.

[3] R. Schreier, "An empirical study of high-order single-bil delta-sigma modulators," IEEE Trans. Circuits Syst. 1/. vol. 40. pp. 461-466, Aug 1993.

14] H. Ardalan and J, J. Paulos, "Analysis of nonlinear behavior in dela-sigma modulalors," IEEE Trans. Circuits Syst., vol. CAS-34, pp. 593-603. Jun 1987.

[5] N. A. Fraser and B. Nowrouzian, "Stability analysis of multiple-feedhach oversampled L- $\triangle$ AD converter configurations." in Proc, IEEE Midwest Symp. Circuits Syst., 20(X), vnl. 2, pp. 676-679.

[6] H. Wang. "A geometric view of sigma delta modulations." IEEE Trans. Circuits Syst. II, vol. 39, pp. 402-405, Jun 1992.

17] __ "On the stability of third-order sigma-delta moduation" in Proc.

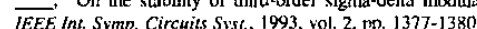

[8] R. Farrell and $O$. Feely, "Bounding the integralor oulpuls of sccond-order sigma-delta modulators," IEEE Trans. Circuits Sy.st. II, vol. 45, pp. 691sigma-delta modun 1998 .

[9] O. Feely. "A tutorial introduction to mon-linear dynamics and chaos and their application to sigma-delta modulators," in In. J. Cirtuit Theory and Applications, 25. 1997, pp. 347-367.

[10] P. Steiner and W. Yang, "A framework for analysis of high-order sigmadelia modulators," IEEE Trans. Circuits Svst. I1. vol. 44. pp. 1-10. Jan 1997.

[11] N. Wong and T. S. Ng, "A generalized direct-lomn delia-operator-based UR fitter with minimum noise gain and sensitivity," IEEE Trans. Circuits Syst, II., vol. 48, no. 4. ๆp. 425-431, Apr 2001.

112] _... "DC slability analysis of high-order, lowpass sigma-delta modulators with distinct NTF eeros," to he suhmitted to IEEE Trans. Circuits Sy.st. "I. 